INPLASY

PROTOCOL

To cite: Hu et al. The mental problems of Chinese medical staff during the COVID-19 outbreak: A meta-analysis. Inplasy protocol 202130112. doi:

10.37766/inplasy2021.3.0112

Received: 30 March 2021

Published: 31 March 2021

Corresponding author:

Ying Li

liying@bch.com.cn

Author Affiliation:

Department of Psychiatry, Beijing Children's Hospital, Capital Medical University, National Center for Children's Health.

Support: Nothing.

Review Stage at time of this submission: Data analysis.

Conflicts of interest:

None declared.

\section{The mental problems of Chinese medical staff during the COVID-19 outbreak: A meta-analysis}

Hu, N1; Deng, H²; He, S3; Chai, J4; Liu, F5; Xiao, X6; Li, Y7.

Review question / Objective: During the outbreak of COVID-19, medical staff were prone to a number of psychological problems. Our study will explore the mental health status of medical staff in China during COVID-19 in a multidimensional manner through meta-analysis.

Condition being studied: Several studies have attempted to investigate the mental health status of medical staff during COVID-19, but their sample sizes were limited and we still lack some multidimensional assessments.

Information sources: Two independent reviewers will conduct comprehensively searches in PubMed, EMBASE.com, Web of Science, Chinese biomedical literature database (CBM), Chinese National Knowledge Infrastructure (CNKI) and Wan fang Database. Languages of the publications will be limited to Chinese and English.

INPLASY registration number: This protocol was registered with the International Platform of Registered Systematic Review and Meta-Analysis Protocols (INPLASY) on 31 March 2021 and was last updated on 31 March 2021 (registration number INPLASY202130112).

\section{INTRODUCTION}

Review question / Objective: During the outbreak of COVID-19, medical staff were prone to a number of psychological problems. Our study will explore the mental health status of medical staff in China during COVID-19 in a multidimensional manner through meta-analysis.

Condition being studied: Several studies have attempted to investigate the mental health status of medical staff during COVID-19, but their sample sizes were 
limited and we still lack some multidimensional assessments.

\section{METHODS}

Search strategy: Retrieval word: "COVID-19" or " 2019 novel coronavirus infection" or "SARS-CoV-2 infection" \& "mental health" or "depression" or "anxiety" or "stress" or "PTSD" or " sleep" \& "medical staff" or "medical personnel" or "health care workers " \& " China".

Participant or population: Chinese medical staff working in hospitals during COVID-19.

Intervention: Not applicable.

Comparator: Not applicable.

Study designs to be included: Cross sectional study.

Eligibility criteria: Inclusion criteria: (1) The study involved Chinese medical staff working in hospitals during COVID-19. (2) The symptoms measured by validated scales. (3) The study gave detection rates for anxiety, depression, sleep disorders or stress-related disorders. (4) Published in Chinese and English. Exclusion criteria: (1) Detection rates for symptoms (anxiety, depression, sleep disorders or stressrelated disorders) were not provided. (2) Articles with duplicate records. (3) The sample size was less than 100. (4) Conference abstracts, news reports, reviews, expert comments, case reports and other literature.

Information sources: Two independent reviewers will conduct comprehensively searches in PubMed, EMBASE.com, Web of Science, Chinese biomedical literature database (CBM), Chinese National Knowledge Infrastructure (CNKI) and Wan fang Database. Languages of the publications will be limited to Chinese and English.

Main outcome(s): Detection rates of depression, anxiety, sleep disorders, and stress-related disorders among Chinese medical staff during COVID-19; differences in mental health status between front-line and non front-line medical staff; the influence of factors such as location and gender on mental health.

Quality assessment / Risk of bias analysis: The quality of each study was assessed by the modified Jadad scale. Two authors independently scored each included trial, and then discussed to reach consensus on any differences. Sensitivity analysis was performed to demonstrate that our statistical analysis was robust. The funnel plot method was used to examine the publication bias to verify whether the results of our study were reliable.

Strategy of data synthesis: All of the analyses were performed in $\mathbf{R}$ (version 3.5.3) using the "meta" or "metafor" packages.

Subgroup analysis: The main results were subgroup analyzed by location, gender and whether they were front-line medical staff.

Sensitivity analysis: Sensitivity analyses will be conducted by excluding studies with high risk of bias to assess the stability of results.

Language: The language is limited to English and Chinese.

\section{Country(ies) involved: China.}

Keywords: COVID-19; medical staff; mental problem; meta-analysis.

Contributions of each author:

Author 1 - $\mathrm{Na} \mathrm{Hu}$.

Author 2 - Hu Deng.

Author 3 - Sushuang He.

Author 4 - Jiabao Chai.

Author 5 - Fuquan Liu.

Author 6 - Xue Xiao.

Author 7 - Ying Li. 